OAI-PMH: http://www.indteca.com/ojs/index.php/Revista Scientific/oai

\title{
Promoción de la Conciencia Ambientalista desde la Perspectiva Axiológica en la Escuela - Comunidad
}

\author{
Autora: Angélica Yaritza Contreras Vega \\ Universidad Pedagógica Experimental Libertador, UPEL \\ angelicac2010@gmail.com \\ Barinas, Venezuela
}

\section{Resumen}

El objetivo del presente estudio es desarrollar acciones de conciencia ambientalista desde la perspectiva axiológica en la escuela - comunidad de Banco Alto, Municipio Pedraza Estado Barinas. La misma estará enmarcada en una investigación acción con sus respectivas fases: diagnóstico, planificación, ejecución, evaluación y sistematización. Los informantes claves del estudio serán 18 estudiantes y 15 miembros de la comunidad. Las técnicas empleadas en esta investigación para recopilar la información corresponderán a la observación participante y la entrevista semi estructurada. Los instrumentos utilizados para reflejar la información serán los registros diarios y la guía de entrevista. Para llevar a cabo la validación de la información se tomará en cuenta los métodos de triangulación. Una vez aplicados los instrumentos, se organizará la información a través de la categorización, teorización, clasificación, síntesis y comparación de esta con el fin de obtener una visión completa en lo posible de la realidad del problema en estudio.

Palabras clave: conciencia ambientalista; perspectiva axiológica;
nidad. \section{comunidad.}




\title{
Promotion of Environmental Consciousness from the Axiological Perspective at School - Community
}

(Project execution)

\begin{abstract}
The objective of this study is to develop environmental awareness actions from the axiological perspective in the school - community of Banco Alto, Pedraza State Barinas State. It will be framed in an action research with its respective phases: diagnosis, planning, execution, evaluation and systematization. The key informants in the study will be 18 students and 15 members of the community. The techniques used in this research to compile the information will correspond to the participant observation and the semistructured interview. The instruments used to reflect the information will be the daily records and the interview guide. To carry out the validation of the information, the triangulation methods will be taken into account. Once the instruments are applied, the information will be organized through the categorization, theorization, classification, synthesis and comparison of this in order to obtain a complete vision of the reality of the problem under study.
\end{abstract}

Keywords: environmental consciousness; axiological perspective; community. 


\section{Introducción}

La formación axiológica para la promoción de valores ambientalistas es un proceso a largo plazo en el cual los individuos se convierten en previsores y participante en el desarrollo del ambiente que los rodea. La concienciación hace despertar a las personas y lo ubica en su realidad, descubriendo todo aquello que afecta en mayor o menor grado la calidad de vida; fomentando el sentido común o un criterio bien formado, haciendo frente a los problemas o poniendo todas sus capacidades al servicio de la naturaleza.

Al respecto, Muñoz (2003) sostiene que: "es evidente que la concienciación ambiental, hoy más que nunca, es un factor decisivo en el proceso de desarrollo sustentable" (pág. 43). En lo que respecta a la problemática ambiental, se hace indispensable en la actualidad tener concienciación ambiental sobre los recursos naturales y la propia realidad social y cultural, que haga posible la utilización inteligente del inmenso potencial de riquezas en beneficio de toda la sociedad.

En tal sentido, la relevancia del estudio radica en ser una herramienta pedagógica que coadyuvará significativamente a promover acciones de conciencia ambientalista desde la perspectiva axiológica en la escuela comunidad de Banco Alto, Municipio Pedraza Estado Barinas. El aporte práctico del estudio se hace presente porque los docentes y demás miembros de la comunidad escolar tendrán a su disposición un material de trabajo que refleja con seriedad, validez y confiabilidad científica la situación ambiental.

En tal sentido, la investigación causará impacto en la institución educativa estudiada y en otras instituciones que se interesen por la temática abordada; porque proporcionará acciones de conciencia ambientalista desde la perspectiva axiológica en la escuela - comunidad de Banco Alto, Municipio Pedraza Estado Barinas.

Al mismo tiempo, la justificación metodológica se hace presente porque aporta un conjunto de acciones de conciencia ambientalista desde la 
perspectiva axiológica en la escuela - comunidad. Todas estas acciones son susceptibles de ser aplicadas, validadas, evaluadas y mejoradas en sus diferentes aspectos. De igual manera, la investigación constituye un proceso de realimentación y aplicación al proceso de la concienciación ambiental.

Desde el punto de vista académico la investigación se enmarca en la línea de investigación estudio de los problemas del medio ambiente, métodos para la protección del ambiente y de los recursos naturales. Esta línea se enfoca en exigir a los distintos actores de la sociedad compromisos y responsabilidades en la aplicación del modelo económico, político, ambiental y social, así como en los patrones de consumo que determinan la calidad de vida.

\section{EI Problema}

En estos tiempos, la educación ambiental como proceso integrador está llamada a generar en la población una concienciación o sensibilización que le permita identificarse con la problemática ambiental tanto a nivel mundial como a nivel especifico, es decir, el entorno donde se vive; para así, identificar las relaciones de interacción e independencia que se dan entre el entorno y el hombre. En tal sentido, la educación ambiental requiere estar dirigida en varias direcciones tomando en cuenta las diferentes características de la población, todos los niveles de educación y también llegar a la población que no asiste a la escuela. A tal efecto, Valdez (2012), señala que la educación ambiental es: Un excelente proceso que, satisfactoriamente enfocado, programado, ejecutado y evaluado en los distintos grupos y sectores de la sociedad puede renovar y estimular los procesos políticos, económicos, sociales y culturales de los países para contribuir a minimizar la pobreza, el analfabetismo, la falta de educación... (pág. 87).

Tal como lo plantea el autor, la formación en el cuidado del ambiente es un excelente recurso para la unificación de esfuerzos en el buen uso y 
aprovechamiento de los recursos naturales que provee el medio. De hecho, es una disciplina que aporta la manera de entender las relaciones entre los conocimientos disciplinares y los problemas ambientales, diferente a la tradicional. En esta se contempla, no solo elementos científicos y tecnológicos, sino también axiológicos que conducen a la promoción de valores como equidad, solidaridad, cooperación, responsabilidad y respeto en el uso de los recursos naturales, la diversidad biológica y cultural.

Desde esta perspectiva, la conciencia ambiental implica cambios de actitud hacia la preservación de los recursos naturales y son los docentes mediante una acción planificada, sistematizada y orientadora los llamados a promover planes o políticas para el logro de una conciencia ambiental que genere en acciones en pro del mejoramiento ambiental. Al respecto, Manes (2010), afirma:

La figura del docente debe promocionar un perfil de capacidad, habilidad, destreza, imagen agradable, responsable, actualizado en los estudios pedagógicos y filosóficos de una política educacional de avanzada que genere en una acción pedagógica escolar cónsona con la realidad educativa (pág. 4).

Lo expresado, por el autor conduce a inferir que los docentes requieren estar actualizados y capacitados en políticas educativas o consustanciados con la problemática ambiental de sus instituciones educativas. Así, la gestión escolar es fundamental, porque es la clave para garantizar el cabal funcionamiento de todos los factores que convergen en la institución y entre ellos la política ambiental. Esto redunda en docentes capacitados, motivados, con significativa vocación de servicio.

En tal sentido, la comunidad Banco Alto del Municipio Pedraza - estado Barinas, no escapa a la realidad antes presentada, porque debido a observaciones informales y por los resultados del Proyecto Educativo Integral Comunitario, se ha podido constatar que no existe en la escuela planes, 
programas o acción educativa direccionada a mejorar la problemática ambiental que se presenta en la comunidad.

Además, la Coordinación de Ambiente no fomenta actividades tendientes a promover valores ambientalistas en la comunidad escolar sobre cómo preservar y aprovechar el ambiente que los rodea. Aunado a lo anterior, en la planificación del Proyecto Educativo Integral Comunitario y el Proyecto de Aprendizajes, los docentes no incluyen o promueven actividades tendientes a tratar la política ambiental. Asimismo, ausencia de investigación que permita establecer la relevancia y pertinencia que tiene la comunidad hacia su escuela.

Lo anterior tiene como consecuencia que las áreas verdes de la escuela están deterioradas, los estudiantes no están siendo concienciados a cuidar y preservar el ambiente. Por su parte, los padres y representantes no se muestran sensibilizados ante la problemática ambiental.

En virtud de lo expresado, se genera la motivación de la autora del estudio en proponer acciones de conciencia ambientalista desde la perspectiva axiológica en la escuela - comunidad de Banco Alto, Municipio Pedraza Estado Barinas; de manera tal, que coadyuve a brindar acciones, recursos y herramientas estratégicas que favorezcan la gestión educativa de las institución objeto de estudio y la incorporación de los docentes, padres, representantes y comunidad en general hacia el aprovechamiento óptimo de los recursos ambientales desde la perspectiva axiológica.

Ante estas realidades planteadas, sería importante considerar las siguientes interrogantes:

¿Qué visón axiológica presenta la escuela y comunidad de Banco Alto en cuanto a la promoción de la conciencia ambientalista?

¿Qué aspectos deben considerarse para diseñar acciones para el desarrollo de la conciencia ambientalista desde la perspectiva axiológica? 
¿Cómo se desarrollarían las acciones axiológicas en cuanto a la conciencia ambientalista de la escuela - comunidad de banco alto?

¿Cuál sería el impacto en la escuela - comunidad de banco alto sobre la promoción de la conciencia ambientalista desde la perspectiva axiológica?

\subsection{Objetivos de la Investigación}

\subsubsection{Objetivo General:}

Desarrollar acciones de conciencia ambientalista desde la perspectiva axiológica en la escuela - comunidad de Banco Alto, Municipio Pedraza Estado Barinas.

\section{Marco Referencial}

El marco referencial constituye las teorías, conceptualizaciones, investigaciones previas y antecedentes en general para la sustentación de la investigación. Sandín (2008), explica que: "es la complejidad de las relaciones que se establecen entre principio filosóficos y ontológicos y sus derivaciones epistemológicas se refleja en las diversas tradiciones o perspectivas teóricas existentes en el ámbito de las ciencias humanas y sociales" (pág. 49). Por tanto, a continuación se desarrolla este proceso de análisis e interpretación de ideas para lograr una visión compleja de la realidad estudiada.

\subsection{Antecedentes de la Investigación}

Se inicia con Bazán (2011), quien ejecutó un Plan Estratégico de Sensibilización Ambiental para la Gestión Comunitaria en el Asentamiento Urbano no Planificado la Floresta de la Parroquia El Carmen del municipio Barinas estado Barinas. La presente investigación tuvo como objetivo proponer un plan estratégico de sensibilización ambiental para la gestión comunitaria del asentamiento urbano no planificado "La Floresta". En 
consecuencia, el estudio se ubica en el paradigma cuantitativo porque el propósito de la misma es la medición estadística de la gestión comunitaria en los habitantes del asentamiento urbano no planificado.

El estudio responde a la modalidad de Proyecto Factible y se estructuró en tres fases: Diagnóstico, Factibilidad y la Propuesta. Es de tipo descriptivo con diseño de campo porque los datos fueron recabados directamente de la realidad objeto de estudio.

También, Pernía (2012), desarrolló una investigación titulada "La Educación Ambiental en la Promoción de Valores Ecológicos en los Docentes del Centro de Educación Inicial Bolivariano Pueblo Nuevo municipio Antonio José de Sucre estado Barinas". El estudio tuvo como propósito analizar la importancia de la educación ambiental en la promoción de valores ecológicos en los docentes del Centro de Educación Inicial Bolivariano "pueblo Nuevo" municipio Antonio José de Sucre estado Barinas.

\subsection{Bases Teóricas}

Parafraseando a Balestrini (2006), se deduce que las bases teóricas es la sección que permite organizar y orientar el sentido de la investigación. Por tanto, las bases teóricas constituyen el fundamento epistemológico del estudio.

\subsubsection{Conciencia Ambientalista}

La conciencia ambiental es la mentalidad que se tiene sobre el impacto de la acción humana en el ambiente. Es decir, entender cómo influyen o repercuten las acciones de cada día de los individuos en el ambiente y cómo esto afecta el futuro del planeta. Parafraseando a Jaula (2002) el término conciencia ambiental está conformado por Conciencia que proviene del término en latín conscientia, y se define como el conocimiento que el ser humano tiene de sí mismo y de su entorno; y ambiente entendido como el entorno o suma total de aquello que rodea, afecta y condiciona a los individuos. 


\subsubsection{La Axiología}

La axiología es la rama de la filosofía que se encarga de estudiar la naturaleza de los valores. Odreman (1998), explica que de allí surge la importancia de su aplicación en los procesos educativos. También, aporta el autor citado que es definida como una ciencia que se encarga del estudio de los valores, destacando la existencia de valores positivos y negativos; 0 valores y desvalores.

Así, su origen, se remonta al año 1902 cuando Paul Laupie la introdujo por vez primera y luego Eduard Von Hartman en 1908. Parafraseando a Bianco (2002), la axiología se divide en dos ramas: la ética, que se refiere a la teoría de los valores morales (lo bueno) y la estética, que está muy relacionada con los valores artísticos (lo bello). Por lo que se puede afirmar que la axiología es un sistema formal para identificar y medir los valores; es la estructura de valores de una persona la que le brinda su personalidad, sus percepciones y decisiones.

En tal sentido, etimológicamente la palabra "ética" viene del griego "ethos", que significa "costumbre", mientras que la palabra moral tiene su origen en el latín "moris" que también significa "costumbre". Por lo tanto, la "ética" y la "moral" están muy relacionadas con las costumbres. De allí, se puede definir la ética como la ciencia de las costumbres, resaltando el estudio de la bondad o maldad en los seres humanos.

Desde esta óptica, los valores éticos y morales dan significado a la existencia del ser humano y crean las condiciones para que los individuos aprendan a hacerse cargo del mundo en que están, asumir las consecuencias de sus acciones y de los efectos que producen en los demás y, reconocer que son autores de sus comportamientos. Así, desde tiempos remotos y antes de que los valores hayan sido objeto de estudio de alguna ciencia, el hombre, tal como lo expone Rivera (2011), "ha establecido valores éticos y morales para actuar en sociedad conforme con las expresiones y costumbres, que varían de 
acuerdo al tiempo, el espacio geográfico o las circunstancias en que estos se desarrollen" (pág. 66). Antes de ahondar sobre los valores éticos y morales, se dilucidará qué se entiende por "moral" y qué se entiende por "ética".

\subsubsection{Rol de la Institución Educativa en la Formación en Valores}

La escuela como institución puede actuar a diferentes niveles para favorecer la conciencia ambiental. Al respecto, Martínez y Galíndez (2005) señala las escuelas pueden actuar en los aspectos siguientes: Favoreciendo la comunicación entre la familia y los profesores, de forma que tanto los pedagogos como las familias tengan a su disposición toda la información y ayuda necesaria para trabajar por el éxito del estudiante, independientemente de que existan o no problemas en los hijos. Ayudando a las familias a establecer un entorno familiar que apoye el aprendizaje.

Por tanto, los padres son los primeros y más importantes docentes para sus hijos, son la clave de su éxito en la escuela y más allá. Por tanto, el apoyo a los padres desde la escuela es un componente clave. Esto implica establecer una plataforma desde la que intercambiar ideas y experiencias sobre el desarrollo físico, intelectual y personal de los niños y adolescentes.

\subsection{Bases Legales}

El marco legal ambiental en Venezuela es bastante amplio por el alcance de sus normativas. De ahí, que la presente investigación se sustenta en la Constitución de la República Bolivariana de Venezuela CRBV (1999), la cual dedica todo un capítulo a los derechos ambientales (capitulo IX) en tal sentido en su artículo 127, contempla el derecho y el deber de cada generación de proteger y mantener el ambiente en beneficio de sí misma y del mundo futuro. Toda persona tiene derecho individual y colectivamente a disfrutar de una vida y de un ambiente seguro, sano y ecológicamente equilibrado; el Estado tendrá el deber de proteger el ambiente, la diversidad biológica, los 
recursos genéticos, los procesos ecológicos, los parques nacionales y monumentos naturales y demás áreas de especial importancia ecológica.

Igualmente, el Estado Venezolano junto con la activa participación de la sociedad civil, deben garantizar un ambiente libre de contaminación y la protección especial de los recursos naturales. Por otra parte, la Constitución en el artículo 107 establece que la educación ambiental es obligatoria en todos los niveles y modalidades del sistema educativo, así como en la educación ciudadana no formal. Este artículo formaliza la universalidad de la educación ambiental, debido a que se especifica como sujeto a ser educado ambientalmente, no sólo a los participantes del sistema escolarizado, sino a todos los venezolanos de toda condición y en todas las circunstancias de enseñanza y aprendizaje.

Así mismo, la nueva doctrina constitucional promueve y establece la participación ciudadana como política de estado en todos los ámbitos sociales y en especial en el de la gestión ambiental. A su vez, la Ley Orgánica del Ambiente (2007), tiene por objeto establecer las disposiciones y desarrollar los principios rectores para la gestión del ambiente en el marco del desarrollo sustentable como derecho y deber fundamental del Estado y de la sociedad, para contribuir a la seguridad del Estado y al logro del máximo bienestar de la población y al sostenimiento del planeta en interés de la humanidad. De igual forma establece las normas que desarrollan las garantías y derechos constitucionales a un ambiente seguro, sano y ecológicamente equilibrado.

\section{Marco Metodológico}

\subsection{Naturaleza de la Investigación}

La investigación se encuentra inserta dentro de un estudio cualitativo, que es definida por Martínez (2004), como "un proceso mediante el cual se construye inductivamente la realidad" (pág. 21). En este sentido, lo que plantea el autor, es que este tipo de estudio proceden de lo social; razón por lo cual 
estudia los fenómenos que son analizados por interrelaciones humanas por medio de la descripción de los hechos para la construcción de conocimientos.

En este contexto, Veliz (2008), señala que la investigación cualitativa "busca comprender los fenómenos desde la perspectiva de los participantes, donde el investigador es el instrumento primario para la recolección de la información y su análisis, partiendo de la observación directa de la realidad social" (pág. 153), por medio de las percepciones de los involucrados en el contexto de la escuela - comunidad de Banco Alto, Municipio Pedraza Estado Barinas.

\subsection{Tipo de Investigación}

En razón de lo planteado, el estudio tendrá como objetivo desarrollar acciones de conciencia ambientalista desde la perspectiva axiológica en la escuela - comunidad de Banco Alto, Municipio Pedraza Estado Barinas, la cual se desarrolló con el apoyo del método de una investigación acción participativa. Sobre el particular, Salazar citado en Suárez (2006), la define como aquella que se encuentra "íntimamente unida a los proyectos sociales que no son otra cosa que el desarrollo de modelos alternativos de planificación, ejecución y evaluación de procesos de transformación social donde ocurre la participación colectiva en la investigación" (pág. 7).

Los planteamientos del autor, se orientan hacia una investigación que se ajusta a una institución educativa por medio del análisis e interpretación de la realidad vivenciada por el investigador, la cual cumple con las fases de diagnóstico, planificación, ejecución, evaluación y sistematización, que son explicadas en los párrafos siguientes.

En la fase del diagnóstico, se realizará la observación de la realidad para identificar e interpretar los fenómenos o problemas, partiendo de la descripción y la explicación de los hechos. De allí, que Contreras (2005), señala que es importante definir lo que se desea cambiar de las situaciones 
referentes, "intentando comprender la naturaleza del problema, aceptando que no se conoce la realidad en profundidad y manteniendo la idea de que los problemas establecidos en el inicio no constituyen el área definitiva de la investigación" (pág. 5).

Por medio de la observación se acopiará información la cual será acumulada en un diario que recoja la vivencia, que posteriormente se explicará. Durante la fase de planificación, se realizará una clasificación y jerarquización de los problemas para ser discutidos de manera colectiva y así tomar decisiones colegiadas para su solución a través de un plan operativo que contendrá actividades y metas, descripción de la población objetivo, definición de los indicadores de éxito, recursos, tiempo y la formulación de compromisos.

A través de la fase de ejecución, se pondrá en marcha la planificación, para lo cual deberá existir un control y evaluación de los procesos realizados y tomar en cuenta los cambios de conducta de los actores y en los procesos, las propuestas se irán modificando y el plan inicial va reestructurándose con la evolución de los eventos. Eisman y Otros (2005) sostiene que: "es la actuación para poner el plan en práctica y la observación de sus efectos en el contexto en que tiene lugar" (pág. 263). Por ende, consistirá en la puesta en práctica de las diversas acciones planificadas, cada una de las cuales se reflejarán en el Capítulo IV del presente estudio. En lo que respecta a la fase de evaluación: Eisman y Otros (ob.cit) la definen como:

En torno a los efectos como base para una nueva planificación. Será preciso un análisis crítico sobre los procesos, problemas y restricciones que se han manifestado y sobre los efectos lo que ayudara a valorar la acción desde lo previsto, deseable y a sugerir un nuevo plan" (pág. 264).

De ahí que esta fase permite verificar y comprobar que los contenidos, estrategias y herramientas que la investigadora programe conjuntamente con 
los informantes, serán trabajados de manera adecuada o efectiva. Finalmente, la fase de Sistematización del Proceso y Experiencias representa el momento de teorización sobre la base de la experiencia.

Eisman y Otros (ob.cit) sostiene que: "la sistematización es la interpretación crítica de una o varias experiencias, que a partir de su reconstrucción y ordenamiento, descubre o explicita la lógica del proceso vivido" (pág. 264). Se inicia con la definición de objetivos, metas de sistematización y descripción de los eventos. Consistirá en ordenar, clasificar y categorizar las ideas, experiencias, interpretaciones y teorías implícitas en las acciones. Esta fase deberá permitir la construcción de conceptos, importación y descubrimiento de teorías y creación de nuevos conocimientos.

\subsection{Informantes}

Por tratarse de una investigación acción participante y ubicarse en el paradigma cualitativo, los informantes claves seleccionados responden a la modalidad intencional o basada en criterios. Según Alanís (2004) afirma que: "la implicación del sujeto investigador es necesaria, ya que facilita la recolección de datos de manera directa; por lo que se requiere un contacto intenso con los sujetos del contexto" (pág. 110); es decir, que se generen lazos de amistad, los cuales llevaron a que se cree un grado de confianza tal que produjo que la información requerida sea más fidedigna y más exhaustiva.

De acuerdo a esta definición, el criterio que orientará la selección de los informantes claves se basará en el hecho de que los mismos pertenecen al contexto estudiado. De allí que la presente investigación contará con la colaboración de 10 estudiantes, 3 miembros de la comunidad, 2 docentes y 3 voceros del consejo comunal de la comunidad Banco Alto, Municipio Pedraza Estado Barinas. 


\subsection{Técnicas e Instrumentos para la Recolección de Datos}

Las técnicas empleadas en esta investigación para recopilar la información corresponderán a la observación participante y la entrevista. Según Eisman y Otros (2005) en la observación participante "el investigador es un ente activo del proceso indagatorio" (pág. 265). Es decir, participa en el contexto en interacción constante e incluso identificándose con los sujetos, pero al mismo tiempo guardando su propio rol de investigador.

En tal sentido, la investigadora se involucrará activamente en el desarrollo de las diversas acciones del estudio, y se integrará de manera armoniosa a las diferentes situaciones y puntos de vista de cada uno de los informantes. Los instrumentos utilizados para reflejar la información suministrada por medio de la observación serán los registros diarios y la guía de entrevista.

Esta entrevista será plasmada mediante una guía de entrevista de preguntas abiertas, que permitirán a los sujetos de estudio, manifestar libremente y en confianza sus conocimientos, ideas, experiencias y dudas con respecto al tema de la investigación.

Bajo esta perspectiva, puede señalarse que la utilización de cada una de estas técnicas implicará un proceso, a través del cual se determinaran las opiniones, intenciones, propósitos y riesgos de todos los implicados a lo largo de la investigación, y que permitirá establecer sus vivencias acerca de cómo influyeron sobre ellos las acciones ejecutadas en el estudio.

\subsection{Validez y Fiabilidad de la Investigación}

Tomando en consideración la investigación acción participante, la validez según Pérez (2008) "concierne a la exactitud con que las conclusiones representen efectivamente la realidad empírica y si los constructos diseñados representan categorías reales de la experiencia humana" (pág. 80). De ahí que 
la validez depende de una actitud de aproximación de igual a igual, entre el evaluador y el evaluado.

Es por ello que para llevar a cabo la validación de las informaciones se tomará en cuenta los métodos de triangulación, a través de los cuales, según Hernández Fernández, y Baptista (2010), expresan que la triangulación es el "hecho de utilizar las diferentes fuentes y métodos de recolección que permite integrar y contrastar toda la información disponible para construir una visión global, exhaustiva y detallada de una experiencia en particular" (pág. 622).

Es decir, se reúnen observaciones e informes sobre una misma situación o sobre algunos aspectos de la misma, efectuados desde distintos ángulos o perspectivas para compararlos y contrastarlos. Es así como se cotejarán las interpretaciones de la investigadora y los sujetos objeto de estudio; se contrastaron las variadas fuentes de información o percepciones de los implicados en el proceso de investigación; además, se valoraron las sugerencias o comentarios, los cuales servirán para profundizar y realimentar el proceso.

Referente a la fiabilidad de este estudio se realizarán observaciones continuas en el aula sobre el interés de los informantes, para detectar debilidades en la aplicabilidad de las acciones que permitan reorientar la concienciación ambiental, los recursos didácticos y la motivación.

\subsection{Técnica de Análisis de la Información Registrada}

Los datos de un análisis cualitativo provienen del trabajo de campo, y se exponen en descripciones que ilustren en el proceso por medio del cual se llega a las estructuras particulares y a la estructura general que los integran. Una vez aplicados los instrumentos, se organizará la información a través de la categorización, clasificación, síntesis y comparación de esta con el fin de obtener una visión completa en lo posible de la realidad del problema en estudio. 
Es por ello que, Tamayo y Tamayo (2008), señalan "el propósito del análisis es resumir las observaciones llevadas a cabo de tal forma que proporcione respuestas a las interrogantes de la investigación". (pág. 40). Es decir, el análisis de la información contempla una etapa de búsqueda sistemática y reflexiva de los datos obtenidos a través de las técnicas y los instrumentos. Bajo esta perspectiva, el análisis se efectuará de forma guiada y ordenada desde el comienzo del estudio y con un carácter flexible.

\section{Conclusiones}

Definitivamente la promoción de acciones ambientalista basada en una educación ambiental que hace reflexión sobre la relación del hombre y la naturaleza; asociada a los valores se puede desarrollar un bienestar entre lo que necesita el hombre de la naturaleza para su bienestar físico y mental, Desde esta óptica, los valores éticos y morales dan significado a la existencia del ser humano y crean las condiciones para que los individuos aprendan a hacerse cargo del mundo en que están, asumir las consecuencias de sus acciones y de los efectos que producen en los demás y, reconocer que son autores de sus comportamientos. En virtud de esta visión se genera la investigación en pro de desarrollar acciones ambientalistas teniendo como base la axiología, para coadyuvar a la escuela comunidad de Banco Alto brindándoles recursos y herramientas estratégicas que favorezcan la gestión educativa para el aprovechamiento óptimo de los recursos ambientales con que cuentan las organizaciones escolares e incorporación de los docentes, padres, representantes y comunidad.

\section{Referencias}

Bazán, M. (2011). Plan Estratégico de Sensibilización Ambiental para la Gestión Comunitaria en el Asentamiento Urbano no Planificado la 
Floresta de la Parroquia el Carmen del Municipio Barinas Estado Barinas. Trabajo UNELLEZ.

Constitución de la República Bolivariana de Venezuela (1999). Gaceta Oficial de la República Bolivariana de Venezuela, 5453, marzo 3.

Jaula, R. (2002). La Conciencia Ambiental: Qué es y Cómo medirla. Trabajo preparado para el IX Congreso Español de Sociología, grupo de trabajo 21: Sociología y Medio Ambiente, septiembre, España.

Jiménez, M. y Lafuente, R. (2007). La Conciencia Ambiental: Sociología y Medio Ambiente, septiembre, España.

Ley Orgánica de Educación. (2009). Gaceta Oficial № 5929 (Extraordinario). Fecha 15 de Agosto de 2009.

Manes, J. (2010). Gestión estratégica para instituciones educativas. Buenos Aires.

Martínez, A. Galíndez, E. (2005). La implicación de los padres en los centros escolares. Psicoteca.

Muñoz, G. y Otros. (2003). Cómo desarrollar competencias investigativas en educación. Colombia: Nomos.

Pernía, L. (2012). La Educación Ambiental en la Promoción de Valores Ecológicos en los Docentes del Centro de Educación Inicial Bolivariano Pueblo Nuevo municipio Antonio José de Sucre estado Barinas. Universidad Fermín Toro.

Rivera, M. (2011). Guía Informativa para docentes: Inducción educativa de valores morales en estudiantes del nivel primario. Escuela Oficial Urbana para Niñas Nro. 97 Monte Verde, jornada Matutina, zona 4 Mixco, Guatemala. Trabajo de Maestría no publicado. Universidad de San Carlos de Guatemala.

Salazar, Y. (2011). Ética profesional. [Documento en línea]. Recuperado de: http://ysabel1408.blogspot.com/2011/02/valores-y-tipos.html 
Educación de la Universidad de Carabobo. Valencia, Venezuela.

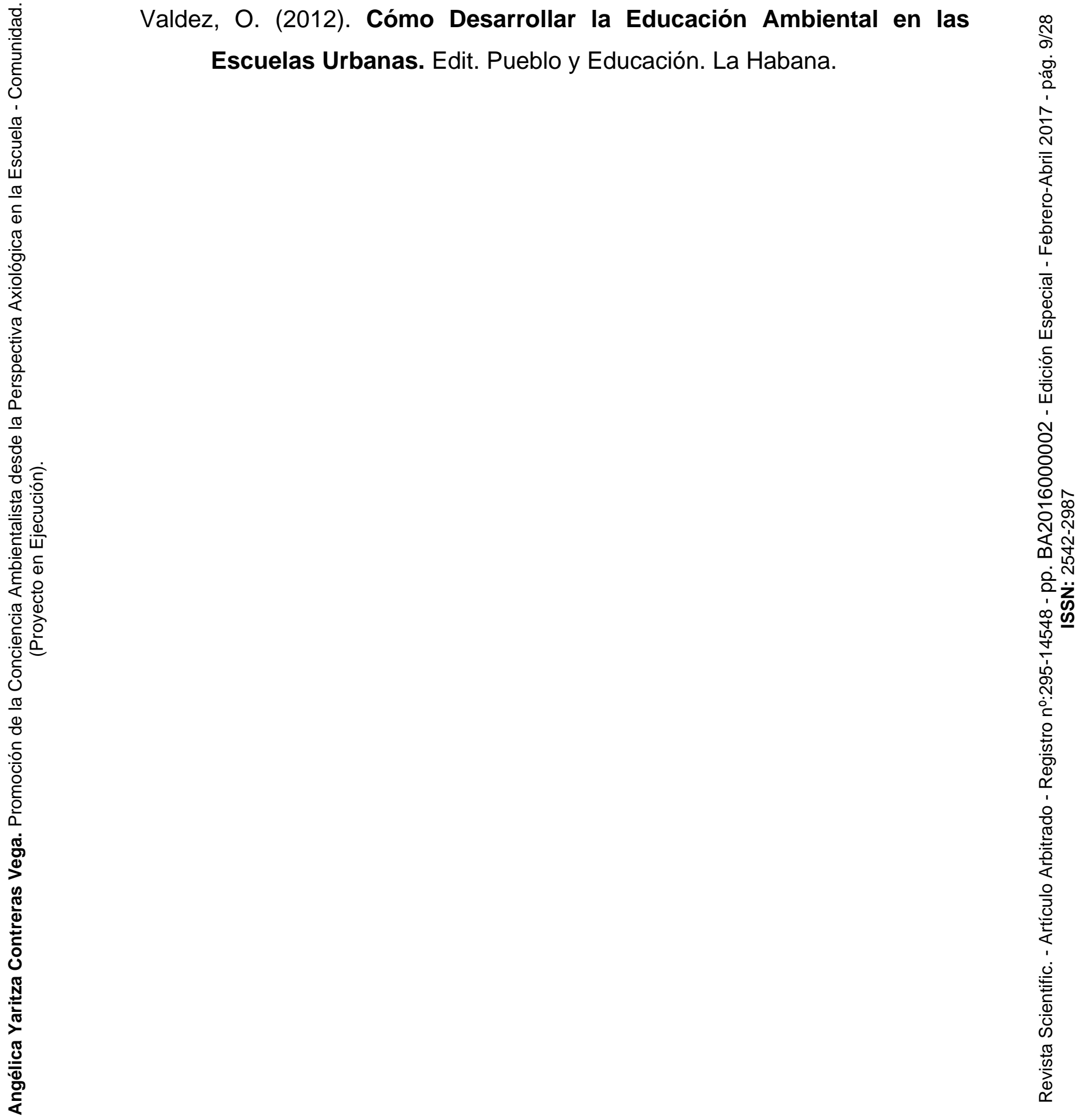




\section{Angélica Yaritza Contreras Vega}

e-mail: angelicac2010@gmail.com

Técnico Superior Universitario en Educación Integral (Instituto Universitario de Tecnología Agustín Codazzi), Licenciada en Educación mención Educación Integral (Universidad Católica Cecilio Acosta) Docente en Ejercicio de Primaria, dependiente del Ministerio de Educación. 